\title{
Efferent Projections of the Mamillary Body in the Cat
}

\author{
By \\ Kahee Niimi, Masahiro Koizuka, Syosuke Kawamura \\ and Kumashige Abe \\ The Third Department of Anatomy, Okayama University Medical School, \\ Okayama, Japan \\ - Received for Publication, February 26, 1972-
}

\section{Introduction}

A considerable amount of research has been reported on the efferent projections of the mamillary body in the cat. This center is said to project to the anterior thalamic nuclei by way of the mamillothalamic tract on the one hand, and to the tegmental nuclei through the mamillotegmental tract on the other. However, the precise topographic organization of efferent fibers from the mamillary nuclei has not been well understood.

In the present study axon degeneration following localized lesions in the mamillary nuclei of the cat was traced in serial sections impregnated by the Nauta-Gygax ('54) silver technique.

The mamillary body is composed of the medial and the lateral nuclei. In the present paper the lateral mamillary nucleus is identical with the nucleus intercalatus in the German literature. The medial mamillary nucleus is divided into two parts: pars medialis and pars lateralis. The pars medialis corresponds to the nucleus magnocellularis of Greving ('25) and to the anterior and medial nuclei of Rose ('39). The pars lateralis is equivalent to the basal and intermediate nuclei of Rose. The basal nucleus corresponds to the nucleus parvocellularis of Greving, while the intermediate nucleus may be comparable to the nucleus mamillaris cinereus of the same author. The terminology employed for dorsal thalamic nuclei is based on that of Niimi and Kuwahara ('72).

\section{Material and Methods}

Operations were performed on 14 adult cats and seven of these, which showed lesions fairly well localized in the mamillary nuclei, were used in the present study. Electrolytic lesions were placed by 
a dorsal approach by means of a stereotaxic apparatus in cats 35,38 , 56,59 and 62 , and manually by a ventral approach in cats 71 and 73 . After a survival period of 7 to 10 days the animals were sacrificed and purfused via the carotid artery with normal saline followed by a solution of $10 \%$ neutral formalin. The brains were removed from the skull, fixed in $10 \%$ neutral formalin, sectioned transversely at $40 \mu$ on the freezing microtome and impregnated by the Nauta-Gygax ('54) silver method.

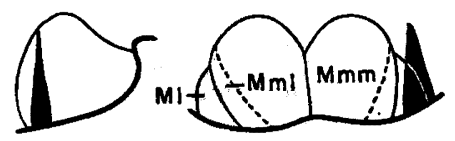

CAT 35
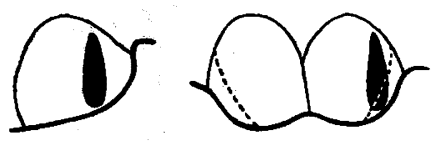

CAT 56
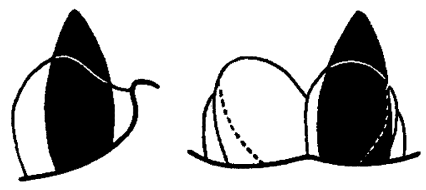

CAT 62
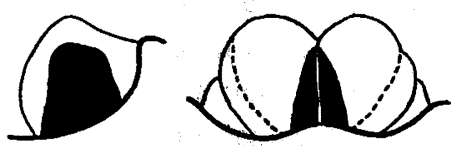

CAT 73
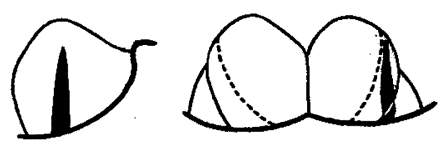

CAT 38
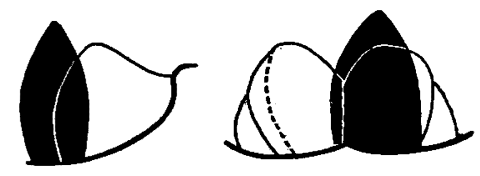

CAT 59
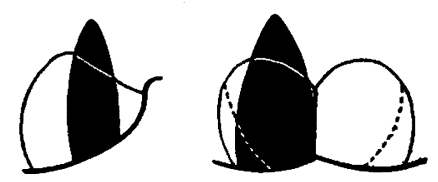

CAT 71

Text-fig. 1. The site and maximal extent of the lesions (black) in the mamillary nuclei in 7 cats. In each case the right figure shows the mamillary lesion in a transverse section, and the left indicates the sagittal extent of the lesion.

\section{Observations}

Cat 35 (Text-fig. 2). The electrolytic lesion was placed in the lateral part of the left lateral mamillary nucleus at anterior levels of the mamillary body and involved the dorsolaterally adjacent white matter. The medial mamillary nucleus was not injured (Text-fig. 1). 
The degenerating fibers in the left mamillothalamic tract pass dorsally through the medial ventral, submedial and anteromedial nuclei of the thalamus and spread into the anterodorsal nucleus from its ventrolateral border. During their course through the medial ventral and submedial nuclei, some fibers appear to terminate in these nuclei. A few fibers are seen to cross the midline diagonally at the dorsal end of the rhomboid nucleus and run along the dorsomedial border of the contralateral anteromedial nucleus to enter the anterodorsal nucleus at its ventromedial extremity.

The anterodorsal nucleus on the side of the lesion shows fairly severe preterminal degeneration throughout most of its extent, containing medium or fine degenerating preterminals. In its ventrolateral part a large number of medium-sized beaded axons are found. Within the anterodorsal nucleus of the opposite side degenerating preterminals are very few in number, and a few medium-sized beads are seen running dorsolaterally. No degenerating fibers are found in its most dorsal part.

The degenerating mamilloseptal fibers pass rostrally through the lateral hypothalamic nucleus as a component of the medial forebrain bundle, though some of them spread out laterally to end in this nucleus. As the preoptic region is reached, they are directed dorsomedially, but a few are found to terminate in the lateral preoptic nucleus. At caudal levels of the septum pellucidum they pass dorsomedially through the diagonal band of Broca and ascend in front of the fornix to enter the medial septal nucleus. In the ventral part of this nucleus fine degenerating beaded axons are seen running dorsally, while in the dorsal part degenerating preterminals predominate. Since the lesion extends somewhat dorsally beyond the lateral mamillary nucleus in this case, the possibility cannot be excluded that the fibers from the mesencephalic tegmentum to the medial septal nucleus might be destroyed. However, the degenerating fibers found in the medial septal nucleus can be regarded at least in part as coming from the lateral mamillary nucleus.

The degenerating fibers of the mamillotegmental tract are assembled dorsolateral to the mamillary body and run caudally, ventral to the red nucleus. They gradually decrease in number and move somewhat medially to lie at first dorsal to the mamillary peduncle and then just lateral to the interpeduncular nucleus. At levels of the commissure of the inferior colliculus they are directed dorsally to form a loose bundle extending along the median raphe. Some of them run further dorsally between the median raphe and the medial longitudinal fasciculus and end in the central gray of the mesencephalon. 
At the most caudal level of the interpeduncular nucleus a small number of fibers diverge from the main bundle of mamillotegmental fibers. They course ventrally just ventrolateral to the interpeduncular nucleus and enter the medial part of the nucleus reticularis tegmenti pontis (Bechterew). The majority are found to end in this nucleus, but a few pass further ventrally to the rostral half of the medial pontine nucleus.

Although a few fibers spread out laterally into the reticular formation, the main bundle of mamillotegmental fibers is directed dorsally to the ventral tegmental nucleus. A fair number of fibers terminate in this nucleus, but others pass dorsally through or by it to ond in the dorsal tegmental nucleus. It is of interest to note that a very few fibers having passed through the ventral tegmental nucleus take a dorsolateral course to end in the nucleus laterodorsalis tegmenti (Castaldi).

Within the ventral tegmental nucleus fine degenerating axons and preterminals are scattered throughout its entire extent, but they are fewer in its large-celled part. The dorsal tegmental nucleus shows somewhat more pronounced degeneration than does the ventral nucleus, particularly in its large-celled ventral part and the extreme medial portion of the small-celled dorsal part.

In this case the mamillary peduncle contains a small number of degenerating fibers which come from the mamillary lesion. These fibers run caudally within the mamillary peduncle, and at the most rostral level of the oculomotor roots they begin to spread dorsolaterally and laterally, but their ultimate distribution cannot be determined. At the middle levels of the interpeduncular nucleus they are no longer present.

Some degenerating fibers diverging from the mamillotegmental tract run dorsomedially and then caudodorsally to enter the mesencephalic central gray of the side of the lesion. A few of them are seen passing through the nucleus of Darkschewitsch but do not end in it. They then course caudally through the medial part of the central gray. As the superior colliculus is reached, they can be found also in the lateral part of the central gray. Passing caudally the degenerating fibers decrease in number and spread laterally into the reticular formation. They can be traced as far caudally as the lowest level of the superior colliculus. In this case the lesion is not limited to the lateral mamillary nucleus but involves the adjacent white matter, so that it is difficult to conclude that the degenerating fibers within the central gray originate solely from the lateral mamillary nucleus. However, by a comparison of this case with cats 38,56 and 73 it may be said that the degenerating fibers within the central 
gray arise largely if not entirely from the lateral mamillary nucleus.

Cat 38 (Text-fig. 3). The focal lesion was placed in the pars lateralis of the medial mamillary nucleus and the extreme ventromedial portion of the lateral mamillary nucleus on the left side at levels a little in front of the middle of the anteroposterior extent of the mamillary body. The adjacent white matter was scarcely involved (Text-fig. 1).

The degenerating fibers in the mamillothalamic tract run dorsally through the middle parts of the submedial and medial ventral nuclei of the thalamus, where some fibers appear to terminate. Passing rostrally, they gradually move medially and course dorsally just medial to these nuclei. Then the majority of them sweep dorsolaterally between the anteromedial and the submedial nuclei, and are distributed throughout the entire extent of the pars parvocellularis of the anteroventral nucleus. In this case a very few degenerating
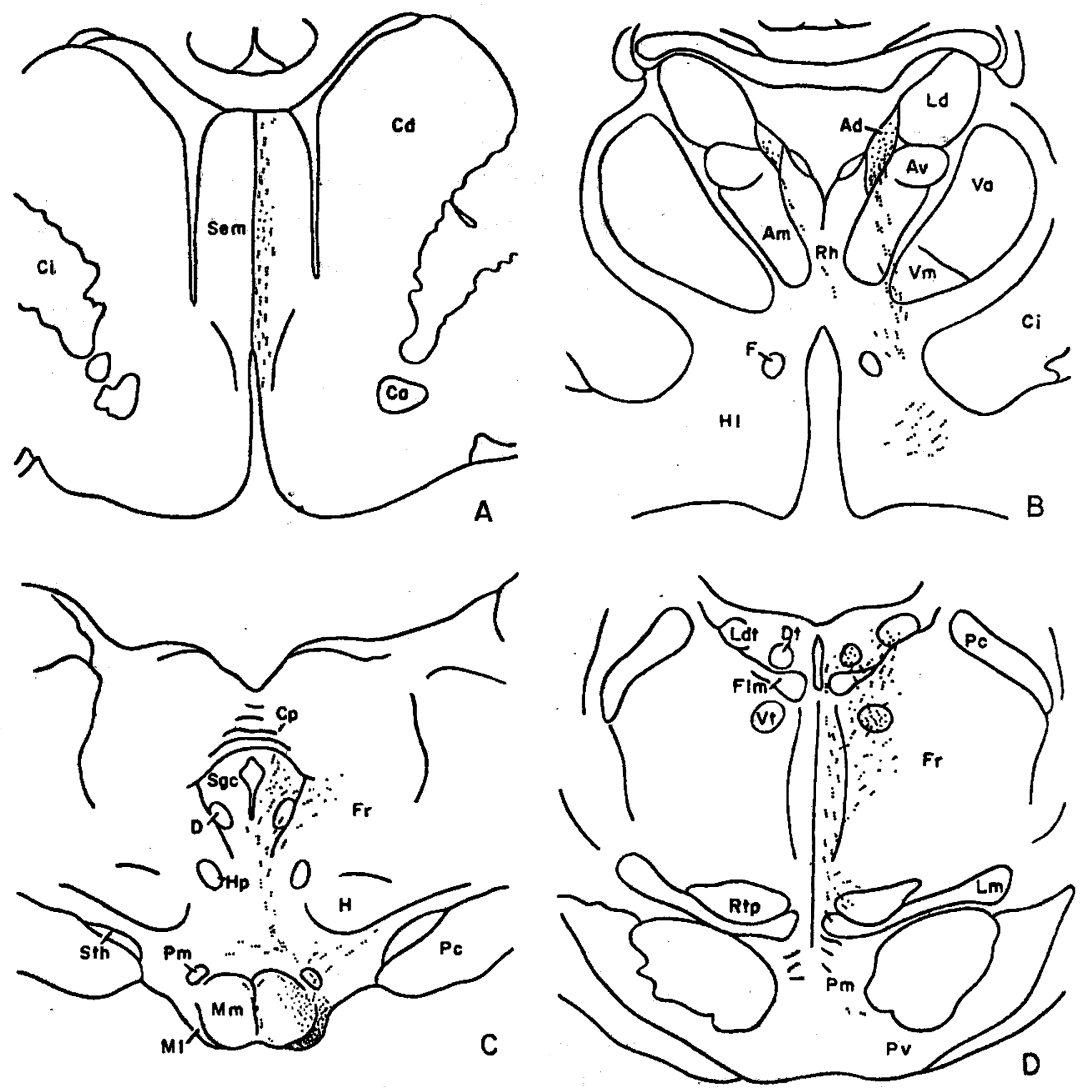

Text-fig. 2. Projection drawings from transverse sections through the brain of cat 35 . In this and subsequent two figures, preterminal degeneration is indicated by dispersed dots and degenerated fibers of passage by rows of dots. 
fibers are found in the ventral and medial parts of the dorsal lateral nucleus, but their exact origin cannot be traced.

On the other hand, some of the degenerating mamillothalamic fibers run dorsally along the dorsomedial border of the anteromedial nuclous, with a fow fibers passing through its ventromedial part, and enter the anterodorsal nucleus at its ventromedial tip. In addition, other fibers take a dorsomedial course to cross the midline in the rhomboid nucleus and pass dorsolaterally along the dorsomedial border of the contralateral anteromedial nucleus into the anterodorsal nucleus.

The anterodorsal nucleus of the operated side undergoes fairly severe preterminal degeneration, particularly in its ventrolateral part. The degenerating fibers in the anterodorsal nucleus of the opposite side are fewer than those of the operated side, but offer essentially the same picture.

The degenerating mamilloseptal fibers are more numerous than in cat 35. Although some fibers spread extensively in the lateral hypothalamic nucleus, the great majority of them run rostrally within the medial forebrain bundle. On reaching the lateral preoptic nucleus, they begin to take a dorsomedial course, but a few fibers appear to terminate there. In more rostral sections they sweep dorsomedially along the fibers of the diagonal band to end in the medial septal nucleus. The degeneration picture in this nucleus is almost the same as that in cat 35 , but differs in that the degenerating fibers are somewhat more numerous.

The degenerating fibers in the mamillotegmental tract pass caudally dorsolateral to the mamillary body. Most of them course along the dorsal border of the mamillary peduncle, though a few fibers extend laterally just ventral to the red nucleus. Approaching the mid inferior collicular levels, they extend dorsally to form an elongated bundle along the median raphe in cross section. At levels of the ventral tegmental nucleus some fibers diverge from this bundle, passing ventrally into the nucleus reticularis tegmenti pontis, and the medial and ventral pontine nuclei. Only a very few fibers can be followed also into the lateral pontine nucleus. The bundle of mamillotegmental fibers, on the other hand, is directed toward the ventral tegmental nucleus, and most of its fibers run dorsally through or by this nucleus to end in the dorsal tegmental nucleus, though a few fibers pass further to the central gray of the pons. It is worth noticing that a very few fibers are seen running dorsally along the median raphe to terminate in the central gray without entering the tegmental nuclei.

Within the ventral tegmental nucleus degenerating beads of axons 
are mainly concentrated in its dorsomedial portion where they can be clearly seen running dorsally. The majority of them are regarded as fibers of passage. In addition, fine degenerating preterminals are also scattered among cells in the ventral nucleus, particularly in its small-celled part. The dorsal tegmental nucleus undergoes conspicuous axon and preterminal degeneration in its large-celled ventral part and the adjacent portion of the small-celled dorsal part.
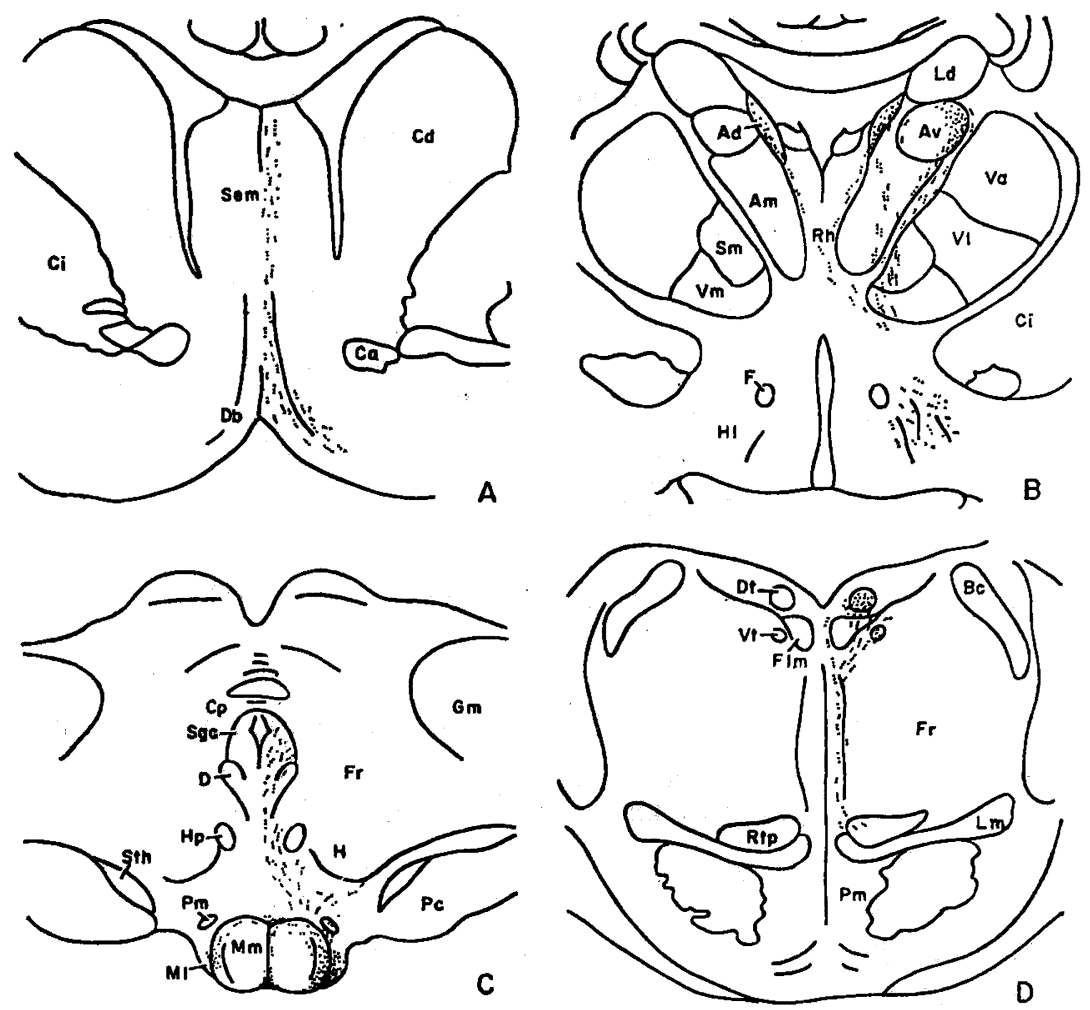

Text-fig. 3. Projection drawings from transverse sections through the brain of cat 38 .

In this case also only a few degenerating fibers are found in the mamillary peduncle.

The fibers entering the mesencephalic central gray are somewhat larger in number than in cat 35. As they descend in the central gray, some fibers spread laterally into the reticular formation of the mesencephalon. At inferior collicular levels no degenerating fibers are seen in the central gray.

Cat 56. The lesion in this cat was limited to the caudal part of the left mamillary body, destroying the lateral portion of the pars medialis and the adjacent small portion of the pars lateralis of the 
medial mamillary nucleus (Text-fig. 1).

The degenerating fibers in the mamillothalamic tract course dorsally through the submedial and the medial ventral nuclei of the thalamus, in which some fibers terminate. At more rostral levels they run dorsally along or through the most medial parts of these nuclei. They then divide into main and accessory bundles. The main bundle sweeps dorsolaterally between the submedial and the anteromedial nuclei, and pass dorsally as well as dorsomedially through the most dorsolateral part of the anteromedial nucleus to end in the dorsolateral portion of the pars magnocellularis and the adjacent portion of the pars parvocellularis of the anteroventral nucleus. Before reaching the anteroventral nucleus some fibers diverge from the main bundle and run dorsomedially through the dorsomedial portion of the anteromedial nucleus to enter the anterodorsal nucleus from its ventrolateral border.

The accessory bundle, after diverging from the main bundle, passes dorsally through or along the most ventromedial portion of the anteromedial nucleus and spreads into the anterodorsal nucleus from its ventrolateral border and ventromedial extremity. However, some fibers course dorsomedially to cross the midline diagonally through the rhomboid nucleus and run along the dorsomedial dorder of the anteromedial nucleus of the opposite side into the ventromedial part of the anterodorsal nucleus at its ventromedial tip.

As in cats 35 and 38 , most of the degenerating mamilloseptal fibers run rostrally through the medial forebrain bundle, though some fibers spread laterally into the lateral hypothalamic nucleus. In the preoptic region they begin to take a dorsomedial course, but practically no fibers terminate there. They then pass dorsomedially by way of the diagonal band to reach the medial septal nucleus. The degenerating fibers of the mamilloseptal tract in this case are fewer in number than those in the above-mentioned cases. A few degenerating fibers are found running through the diagonal band into the medial septal nucleus on the opposite side, but their origin cannot be determined.

The terminal distribution of the mamillotegmental tract is essentially similar to that in cat 38 . At mid inferior collicular levels some degenerating fibers diverge from this tract and pass ventrally to end in the nucleus reticularis tegmenti pontis and the medial and ventral pontine nuclei. The degenerating fibers of the main tract course dorsally and reach the ventral tegmental nucleus to terminate in it, with some fibers passing further to the dorsal tegmental nucleus, particularly to its large-celled ventral part and the adjacent portion of the small-celled dorsal part. A few fibers, however, run through 
the dorsal tegmental nucleus into the central gray of the pons. In this case also, some fibers from the main tract partly pass dorsally along the median raphe to reach the central gray directly, and partly cross the midline to end in the rostral part of the contralateral ventral tegmental nucleus, with a very fow fibers running further through it.

Within the mamillary peduncle a small number of degenerating fibers are seen. At levels of the oculomotor nerve roots most of them leave the mamillary peduncle to run dorsally and appear to join the mamillotegmental tract; some fibers pass laterally along the dorsomedial border of the substantia nigra, though their destination cannot be determined.

The degenerating fibers entering the central gray of the mesencephalon are almost the same as in cats 35 and 38 but somewhat smaller in number.

Cat 59. The electrolytic lesion was fairly extensive and destroyed the rostral one-third of the mamillary body and the dorsal and ventral premamillary nuclei on the left side. The ventromedial hypothalamic nucleus was not injured. In cross sections the lesion was found in the pars medialis and the adjacent portion of the pars lateralis of the left medial mamillary nucleus; extending dorsally to destroy the overlying white matter and involving the medial margin of the right medial mamillary nucleus (Text-fig. 1).

The left mamillothalamic tract is severely affected. The degenerating fibers in this tract pass dorsally through the medial ventral and the submedial nuclei of the thalamus, or along the ventromedial border of the former nucleus, and divide into main and accessory bundles. During their course through the medial ventral and submedial nuclei some fibers terminate in these nuclei. The main bundle passes dorsolaterally between the anteromedial and the submedial nuclei. Some of them spread into the anteromedial nucleus from its ventrolateral border, while the others run further dorsolaterally to enter the anteroventral nucleus from its ventral and ventrolateral margin. In this nucleus degenerated preterminals decrease in number toward the lateral periphery.

The accessory bundle passes dorsally along the ventromedial border of the anteromedial nucleus. Then some of its fibers are directed dorsolaterally to spread into the whole extent of the anterodorsal nucleus from its ventromedial extremity. The others sweep dorsomedially, cross the midline in the rhomboid nucleus and run along the dorsomedial border of the contralateral anteromedial nucleus to enter the anterodorsal nucleus at its ventromedial extremity.

In this case the lesion extends to the medial margin of the right 
medial mamillary nucleus, so that the right mamillothalamic tract contains a fair number of degenerating axons. After passing through the medial ventral and the submedial nuclei, these axons divide into main and accessory bundles. The former runs through the ventromedial extremity of the anteromedial nucleus and then enters the dorsomedial periphery of this nucleus. The latter courses along the ventrolateral border of the anteromedial nucleus and passes through the most dorsolateral part of the anteromedial nucleus to enter the medial portion of the pars magnocellularis of the anteroventral nucleus. In the most caudal portion of the anteroventral nucleus no degenerating fibers are seen.

The degenerating mamilloseptal fibers on the left side are directed rostrally to the septal nuclei; some fibers spread out laterally to the lateral hypothalamic nucleus. On reaching the preoptic region and the diagonal band, many of them sweep dorsomedially to end in the medial septal nucleus. It should be noted that a fair number of them terminate in the lateral septal nucleus which contains very fine degenerating preterminals with an intermingling of some fine chains of beads. This may result from the lesion involving the premamillary nuclei in this case. On the right side also, a small number of degenerating mamilloseptal fibers can be seen. Most of them end in the ventral part of the medial septal nucleus, while a few enter the most caudal part of the lateral septal nucleus. They are considered to come largely from the lesion involving the medial mamillary nucleus and the premamillary nuclei on the right side.

The degenerating fibers of the mamillotegmental tract run caudally, spreading out more or less laterally to lie between the red nucleus and the habenulopeduncular tract. They then accumulate dorsolateral to the interpeduncular nucleus. At caudal levels of the posterior commissure a number of fibers diverge from them and are directed ventrally to end largely in the nucleus reticularis tegmenti pontis and partly in the medial pontine nucleus. The main bundle of the mamillotegmental fibers is directed dorsally to the ventral tegmental nucleus; many fibers end in this nucleus, but some pass through or by it to terminate in the dorsal tegmental nucleus, particularly in the large-celled ventral part, and a few pass further to the central gray of the pons. Within the ventral tegmental nucleus degenerated preterminals are crowded in the small-celled part and are scattered in the large-celled part. It should be mentioned that a fow fibers from the main bundle partly run dorsally along the median raphe into the central gray, and partly cross the midline to end in the ventral tegmental nucleus of the opposite side.

In this case the right mamillotegmental tract is almost free from 
degeneration, though the medial margin of the medial mamillary nucleus is involved in the lesion. This may indicate that this part of the nucleus contributes scarcely any fibers to the mamillotegmental tract, while it sends many fibers into the mamillothalamic tract.

Within the mamillary peduncle a fow degenerating fibers are seen bilaterally, but are sparser on the right side. Their course and termination are similar to those in the above-mentioned cases.

A fair number of degenerating fibers diverging from the left mamillotegmental tract pass dorsally into the mesencephalic central gray. They run caudally through the lateral part of the central gray, but some fibers spread laterally into the reticular formation.

Cat 62. The focal lesion was placed at about the middle of the anteroposterior extent of the left mamillary body. In cross sections it covered almost the whole extent of the medial mamillary nucleus, except its medial and lateral margins, and extended dorsally to destroy the overlying white matter but did not involve the right mamillary nuclei (Text-fig. 1).

The degeneration of the mamillothalamic tract is essentially similar to that in cat 59, but differs in that degenerating fibers are sparse in the medial periphery of the anteroventral and anteromedial nuclei, though they are distributed in the whole extent of these nuclei. In addition, the degenerating fibers in the anterodorsal nuclei of both sides are relatively more abundant than in cat 59. This may be due to the greater involvement of the pars lateralis of the medial mamillary nucleus.

The degenerating mamilloseptal fibers pass rostrally as an ascending component of the medial forebrain bundle, with some fibers spreading laterally to the lateral hypothalamic nucleus and to the lateral preoptic nucleus. They then sweep dorsomedially through the diagonal band and terminate in the medial septal nucleus. A few fibers, however, are directed dorsolaterally to the region just ventral to the globus pallidus. On the contralateral side also, a very few fibers are seen running dorsolaterally through the diagonal band to end in the ventral part of the medial septal nucleus.

The distribution of the degenerating mamillotegmental fibers is essentially the same as that in cat 59. The mamillary peduncle contains only very few degenerating fibers as in the above-mentioned cases.

The fibers contributing to the dorsal longitudinal fasciculus are comparatively large in number in this case. This may be due to the fact that the lesion extends a little more dorsally beyond the mamillary body than in the other cases.

Cat 71 (Text-fig. 4). The lesion, similar in extent to that in cat 

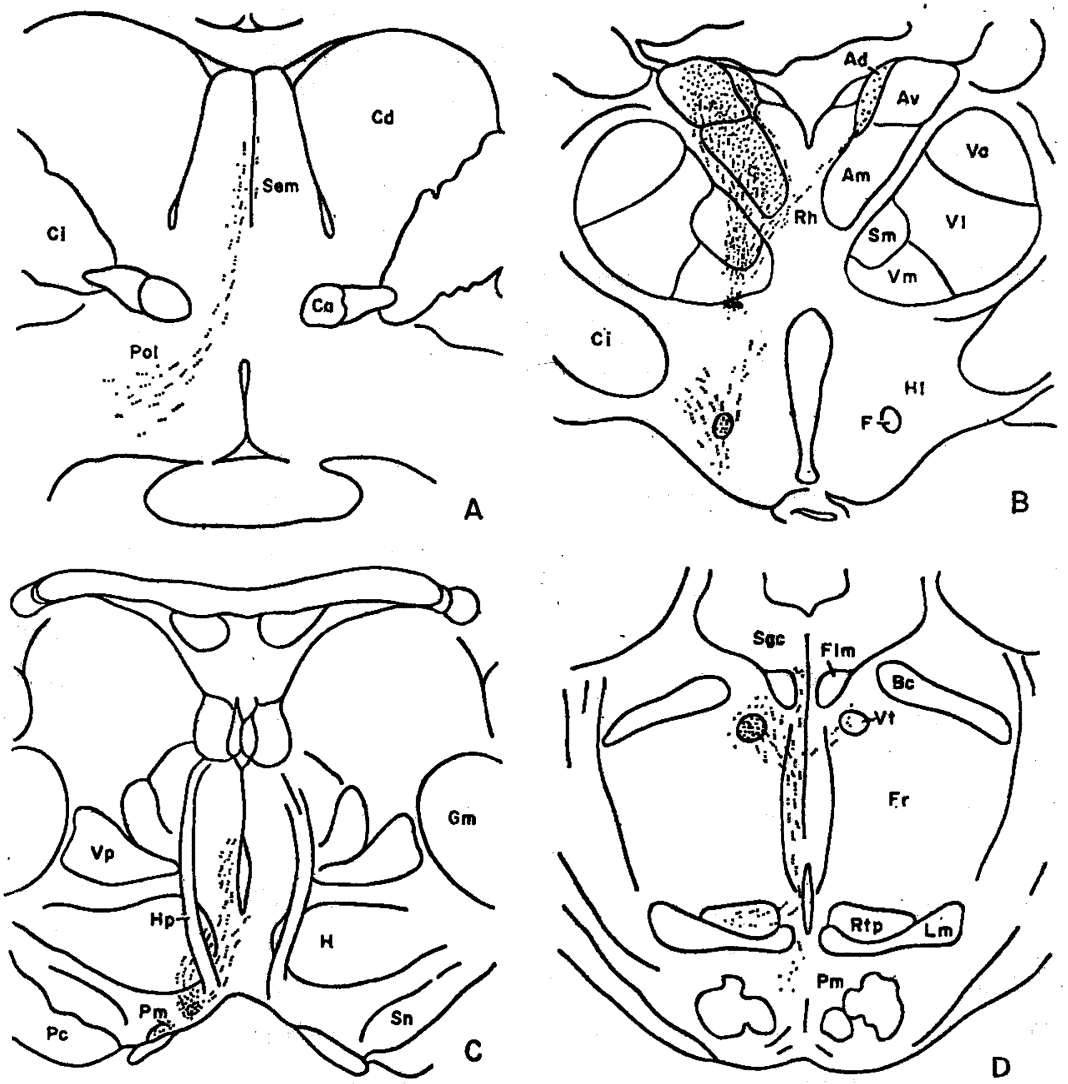

Text-fig. 4. Projection drawings from transverse sections through the brain of cat 71 .

62, was placed manually by a ventral approach in the middle portion of the right mamillary body in anteroposterior extent. In cross sections it covered most of the pars medialis, except for the dorsolateral border and the ventromedial edge of the pars lateralis of the medial mamillary nucleus. It extended dorsally more or less beyond the mamillary body but did not involve the left medial mamillary nucleus (Text-fig. 1).

The distribution of degenerating fibers from the mamillary lesion in this case is similar to that in cat 59. The mamillothalamic tract divides into the main and the accessory bundles before entering the anterior thalamic nuclei. The main bundle divides further into medial and lateral branches. The medial branch spreads into the whole anteromedial nucleus, whereas the lateral branch is distributed throughout the extent of the pars magnocellularis of the anteroventral nucleus. The accessory bundle runs dorsolaterally along the dorso- 
medial border of the anteromedial nucleus to enter the anterodorsal nucleus, but some of its fibers cross the midline to reach the contralateral anterodorsal nucleus.

The course and distribution of the degenerating mamilloseptal fibers are similar to those in cat 62 . However, scarcely any degenerating fibers are found in the course of the mamilloseptal tract on the contralateral side.

The terminal distribution of the mamillotegmental tract is essentially the same as that in cats 59 and 62 , so that it needs no repetition here.

Within the mamillary peduncle a small number of degenerating fibers are found running caudally as in the above-mentioned cases. The degenerating fibers contributing to the dorsal longitudinal fasciculus are somewhat fewer in number than those in cats 59 and 62 , but their course and distribution are similar.

Cat 73. The lesion was made by a ventral approach in the middle portion of the mamillary body in anteroposterior extent. In cross sections it was situated symmetrically in the midline, involving the ventromedial portions of the pars medialis of both medial mamillary nuclei (Text-fig. 1).

In this case the degeneration following the mamillary lesion is found bilaterally and offers the same picture on both sides. The degenerating fibers of the mamillothalamic tract are distributed in the medial part of the anteroventral nucleus and the dorsomedial part of the anteromedial nucleus. No degeneration is found in the anterodorsal nucleus.

The degenerating mamilloseptal fibers course rostrally through the lateral hypothalamus and the diagonal band of Broca and terminate in the medial septal nucleus as in cats 62 and 71 .

Some degenerated fibers from the mamillary lesion are seen to enter the mesencephalic central gray and the mamillary peduncle as in the above-mentioned cases.

Unfortunately, no sections are available caudal to the mid superior collicular level in this case, so that it is impossible to trace the degenerating mamillotegmental fibers to their destination.

\section{Discussion}

The mamillothalamic tract (Text-fig. 5)

There can be no doubt that the pars medialis of the medial mamillary nucleus sends fibers to the ipsilateral anteroventral and anteromedial thalamic nuclei. Our data further reveal that its most lateral portion projects to the dorsolateral portion of the pars magno- 
cellularis of the anteroventral nucleus, while the most medial portion projects to the medial portion of the pars magnocellularis of the anterovental nucleus and the dorsomedial periphery of the anteromedial nucleus. On the other hand, the middle part of the pars medialis is connected with the anteromedial nucleus, except the dorsomedial periphery, and probably with the ventrolateral portion of the pars magnocellularis of the anteroventral nucleus. Available evidence indicates furthermore that the pars lateralis of the medial mamillary nucleus sends fibers to the pars parvocellularis of the ipsilateral anteroventral nucleus and both anterodorsal nuclei, and that the lateral mamillary nucleus gives off fibers only to the anterodorsal nuclei of both sides. It is of interest to note that the pars medialis of the medial mamillary nucleus (nucleus magnocellularis of Greving) projects to the pars magnocellularis of the anteroventral nucleus, whereas the pars lateralis, corresponding largely to the nucleus parvocellularis of Greving, projects to the pars parvocellularis of the anteroventral nucleus.

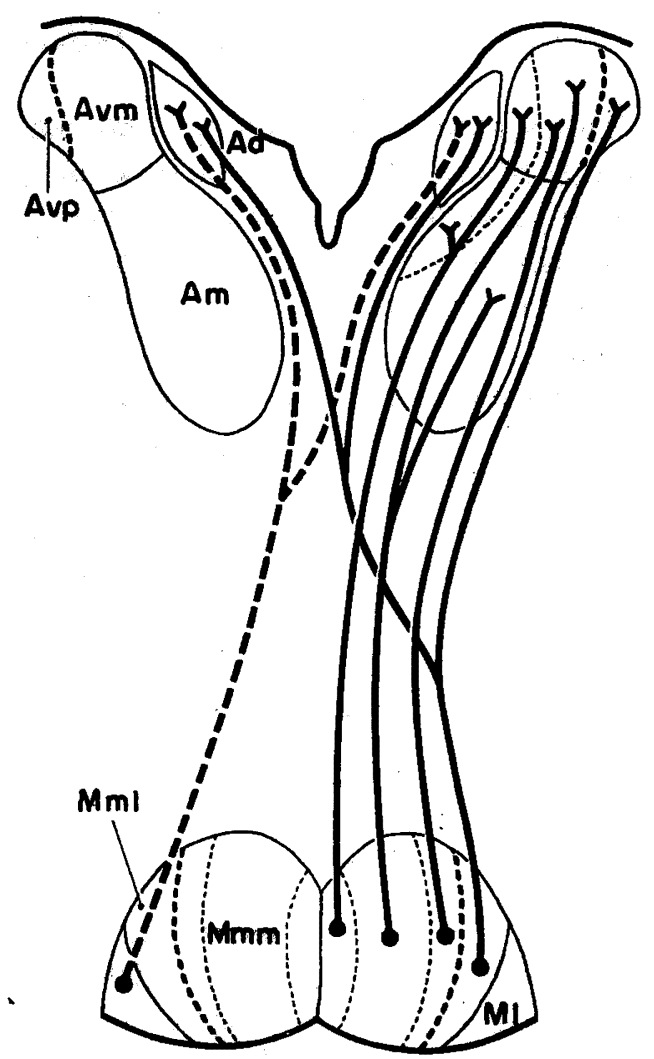

Text-fig. 5. Diagram showing the distribution of mamillothalamic fibers to the anterior thalamic nuclei. 
The precise distribution of the efferents from various parts of the mamillary body has not been sufficiently understood. Clark ('33) studied the mamillothalamic tract in the rat by the Marchi method, and reported that this tract ends in each of the three homolateral anterior thalamic nuclei, and that a number of fibers in the interanterodorsal commissure cross the midline to end in the contralateral anterodorsal nucleus. Powell and Cowan ('54), based on retrograde degeneration study in the rat, revealed that each of the elements in the medial mamillary nucleus has an independent projection to one of the anterior thalamic nuclei; the pars medialis projects to the anteromedial nucleus, the pars posterior to the anteroventral nucleus and the pars lateralis to the anterodorsal nucleus. They also stated that the lateral mamillary nucleus shows no retrograde changes after anterior thalamic lesions. Our observations, however, differ from Powell and Cowan's in that the pars medialis sends fibers to the anteroventral nucleus in addition to the anteromedial nucleus, that the pars lateralis projects not only to the anterodorsal nucleus but also to the pars parvocellularis of the anteroventral nucleus, and that the lateral mamillary nucleus also contributes fibers to the mamillothalamic tract.

Fry et al. ('63) stated that the mamillothalamic tract fibers provide terminations on neurons of the ventromedial nucleus of the thalamus along the intrathalamic course of the tract. Our results also revealed that some of the mamillothalamic fibers terminate in the medial ventral and submedial nuclei, but the majority pass through these nuclei into the anterior nuclei.

Guillery ('57) studied the degeneration following destruction of the mamillary body of the rat by the Nauta-Gygax method. According to him, the mamillothalamic tract can be traced to each of the three ipsilateral anterior thalamic nuclei and to the contralateral anterodorsal nucleus. The pars lateralis of the medial mamillary nucleus appears to have a unilateral projection to the anterodorsal thalamic nucleus, whereas the lateral mamillary nucleus seems to have a bilateral projection to the anterodorsal nuclei. The results of the present study indicate that the pars lateralis of the medial mamillary nucleus, as well as the lateral mamillary nucleus, sends fibers to the anterodorsal nuclei of both sides.

Fry et al. ('63) studied the mamillothalamic tract after destruction of the mamillary nuclei in the cat by the ultrasonic method. They concluded that the medial mamillary nucleus projects to the ipsilateral anteroventral and anteromedial nuclei of the thalamus, while the lateral mamillary nucleus projects bilaterally to the anterodorsal thalamic nucleus. They further suggested that all parts of the 
anteroventral and anteromedial nuclei do not receive equal. projections from all portions of the medial mamillary nucleus. The present investigation reveals the difference in the distribution of the mamillothalamic fibers from different parts of the medial mamillary nucleus, and it is particularly to be noted that in this respect the pars medialis differs markedly from the pars lateralis (the basal and intermediate parts) where the fornix fibers terminate in the rabbit (Sprague and Meyer, '50).

The mamilloseptal tract

In all of our cases the mamilloseptal fibers run rostrally through the medial forebrain bundle, but some fibers spread out laterally to the lateral hypothalamic nucleus. As they reach the preoptic region and the diagonal band, they sweep dorsomedially to end in the medial septal nucleus. In their course a few fibers terminate in the lateral preoptic nucleus, but scarcely any fibers end in the nucleus of the diagonal band. In cat 59 some fibers terminate in the lateral septal nucleus. This may result from inadvertent damage to the dorsal and ventral premamillary nuclei.

Nothing has been found in the literature concerning the precise analysis of the mamillary projection to the septal nuclei. Guillery (57) divided the ascending fibers in the medial forebrain bundle of the rat into two; a medial and a lateral component. According to his results, the medial component ending largely in the medial septal nucleus may originate from the midbrain or pons, while the lateral component which terminates in the lateral septal nucleus may arise from the premamillary hypothalamus. Our results are well in accord with his observation on the lateral component, but not entirely with that on the medial component. Morin ('50) could trace degenerating fibers to the preoptic region following lesions in the caudal hypothalamus, including the mamillary body, of the guinea pig. In most of our cases also the lesions are not confined strictly to the mamillary nuclei. However, the results from the cases with fairly well localized lesions, particularly made by a ventral approach, lead to the conclusion that the degenerating fibers entering the medial septal nucleus arise, at least in part, from the mamillary nuclei. The mamilloseptal tract can mediate impulses from the mamillary nuclei to the septohippocampal tract. Votaw ('60) suggested that a part of at least one neural arc for emotional expression is represented by a pathway that discharges from the septal nuclei to the hippocampi. In connection with this view the presence of the mamilloseptal fibers is significant.

The mamillotegmental tract (Text-fig. 6)

As has been stated above, most fibers of the mamillotegmental tract are distributed to the ventral tegmental nucleus, the dorsal 
tegmental nucleus and the central gray of the pons, while some fibers pass ventrally into the nucleus reticularis tegmenti and the medial, ventral and lateral pontine nuclei. The fibers from the pars medialis of the medial mamillary nucleus enter the ventral tegmental nucleus, particularly the small-celled part, though some fibers pass further to the large-celled part of the dorsal tegmental nucleus. Besides, a few fibers partly pass dorsally along the median raphe into the central gray of the pons, and partly cross the midline to run into or through the small-celled part of the ventral tegmental nucleus of the opposite side. The fibers from the pars lateralis of the medial mamillary nucleus terminate partly in the ventral tegmental nucleus and largely in the dorsal tegmental nucleus, with a few fibers passing further to the central gray of the pons. In addition, a very few fibers can be observed running along the median raphe into the central gray without passing through the tegmental nuclei.

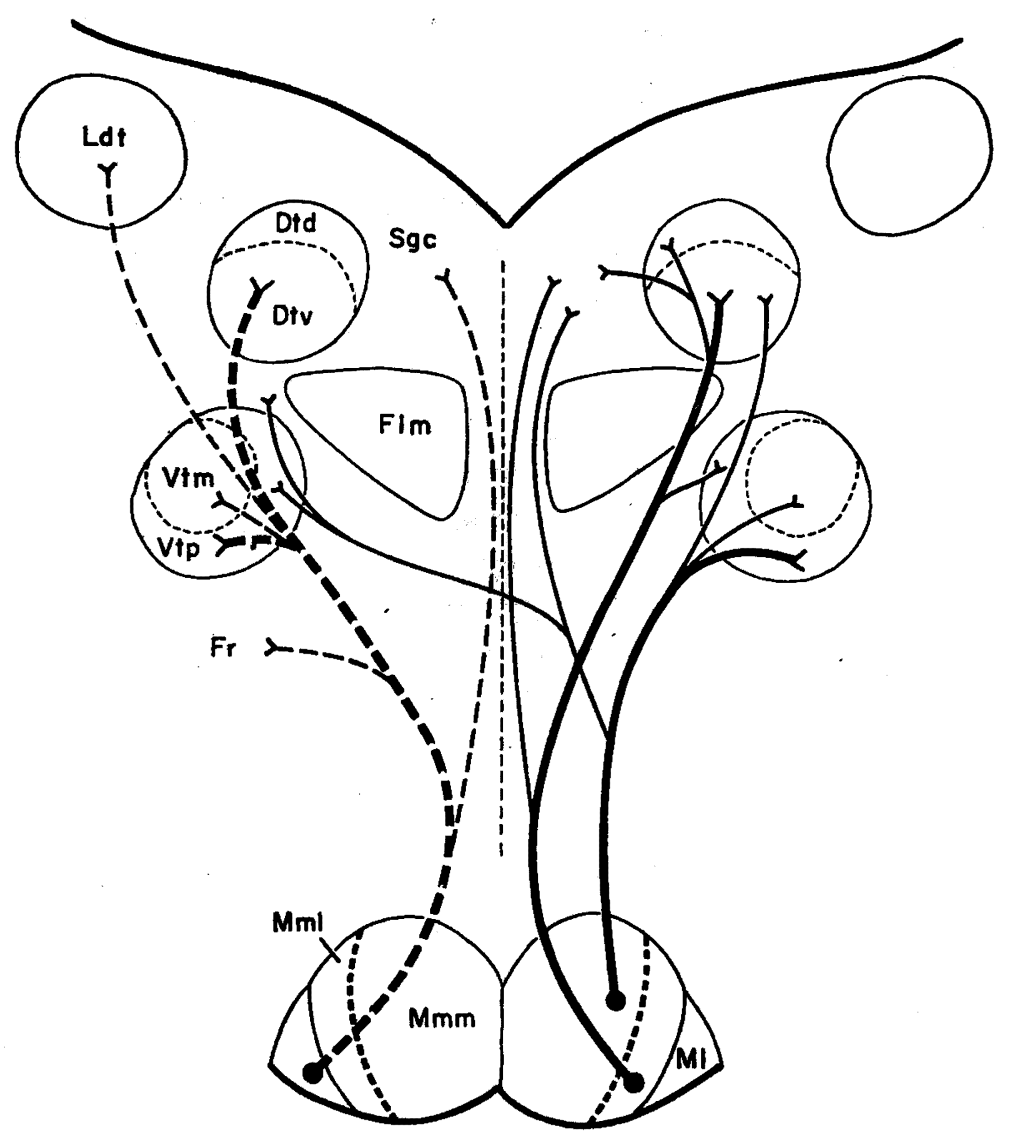

Text-fig. 6. Diagram showing the distribution of mamillotegmental fibers to the tegmental nuclei and the adjacent structures. 
The lateral mamillary nucleus also contributes fibers to the mamillotegmental tract. Most of these fibers are directed to the ventral and dorsal tegmental nuclei to terminate there, while some fibers are distributed to the reticular formation and the tegmental laterodorsal nucleus.

The mamillotegmental tract has long been studied by many authors. Gudden (1881) first described it as "Haubenbündel des Corpus mamillare" and considered it to end among cells of the deep tegmental nucleus (ventral tegmental nucleus). Some authors also traced the mamillotegmental tract to this nucleus (Economo and Karplus, '10 ; van Valkenburg, '12 ; Ibáñez, '35 ; Morin, '50). Yamagata ('27) found degeneration in the deep tegmental nucleus and slight degeneration in the dorsal tegmental nucleus following destruction of the medial mamillary nucleus in the rabbit. Most of the later studies have demonstrated that the mamillotegmental tract terminates in both ventral and dorsal tegmental nuclei (Guillery, '57; Ban and Zyo, '63). Our results confirmed these data and further demonstrated the fibers to the central gray, nucleus reticularis tegmenti pontis, pontine nuclei and the contralateral ventral tegmental nucleus. Thus it is probable that the mamillotegmental tract relays impulses from the mamillary body to the dorsal longitudinal fasciculus directly as well as indirectly, and that the mamillary nuclei are connected with the cerebellum by way of the mamillotegmental and pontocerebellar tracts. Brodal ('54) showed that the tegmental reticular nucleus of the pons also sends its axons to the cerebellum. In accordance with our results, Guillery ('57) traced degenerating mamillotegmental fibers to the tegmental reticular nucleus, in addition to the tegmental nuclei. Ban and Zyo ('63) found Marchi degeneration in the red nucleus following a lesion in the medial mamillary nucleus. However, in none of our cats were degenerating preterminals found in the red nucleus.

Considerable differences of opinion exist with regard to the origin of the mamillotegmental tract. According to Koikegami ('38) this tract arises from the magnocellular nucleus, particularly the anterior part, in the guinea pig. Yamagata ('27) considered it to originate from the medial mamillary nucleus except for the posterior part. Ibáñez ('35) stated that the mamillotegmental tract degenerates also after lesion of the lateral mamillary nucleus and that the degeneration is chiefly contralateral. However, Guillery ('57) found no clear evidence for considerable crossing of the mamillotegmental tracts such as has been described by Ibáñez. Our data reveal that the lateral mamillary nucleus contributes fibers to the mamillotegmental tract, but that these connections are not crossed but are solely ipsilateral. Our results indicate also that the mamillotegmental tract arises from 
all of the mamillary nuclei, except for the extreme medial portion of the medial mamillary nucleus, and shows the precise topographic organization as mentioned above.

There has been much discussion concerning the relationship between the mamillothalamic and mamillotegmental tracts. Cajal ('11) observed a bifurcation of axons from the medial mamillary nucleus into mamillothalamic and mamillotegmental fibers. Van Valkenburg ('12), by contrast, recognized loss of nerve cells in the dorsal part of the medial mamillary nucleus after destruction of the mamillotegmental tract. He noticed the close developmental relations between the mamillothalamic tract and the ventral part of the medial mamillary nucleus, as well as between the mamillotegmental tract and the dorsal part of the medial mamillary nucleus, and suggested that both pathways are independent of each other. Powell and Cown ('54) and Cowan and Powell ('54), using the rat and the rabbit respectively, traced the origins of the mamillothalamic and mamillotegmental tracts by means of the method of retrograde cell degeneration and concluded that the dorsomedial portion of the pars medialis of the medial mamillary nucleus undergoes no degeneration after complete destruction of the anterior thalamic nuclei. However, our data cast serious doubt on the idea that the mamillothalamic and mamillotegmental tracts arise from separate parts of the mamillary body. In all of our cases, where the lesions were placed in various parts of the mamillary body, both pathways are demonstrated. Powell and Cowan suggested that the cells of the dorsomedial third of the pars medialis send their axons in the mamillotegmental tract. In cat 56 of our cases, in which that portion remains intact, the mamillotegmental tract is also degenerated. In cat 59 where the lesion involves the extreme medial portion of the right medial mamillary nucleus, the right mamillothalamic tract is clearly demonstrable, while the right mamillotegmental tract is difficult to trace. Yamagata ('27) reported that the caudal part of the mamillary body contributes no fibers to the mamillotegmental tract. However, in cat 56 of our cases, where the lesion is localized in the caudal part of the mamillary body, the mamillotegmental tract is clearly demonstrated. Recently, Fry ('70) concluded from a quantitative study of the mamillary body that one half the neurons of the medial mamillary nucleus project into the mamillothalamic tract with no branch in the mamillotegmental tract, and that all of the medial mamillary neurons that project to the ventral tegmental nucleus (approximately 11\%) project to the ventral medial nucleus of the thalamus.

The mamillary peduncle

In all of our cases a small number of degenerating fibers are 
found in the mamillary peduncle of the operated side. They descend in the mamillary peduncle, and at levels from the most rostral part of the interpeduncular nucleus to the oculomotor nerve roots, many of them are directed dorsally to join the mamillotegmental tract. Others, however, descend further caudally in the mamillary peduncle and can be traced as far as the middle levels of the interpeduncular nucleus, where they spread laterally along the dorsomedial border of the substantia nigra. Nothing definite can be stated concerning details of origin of the degenerating fibers in the mamillary peduncle. However, these fibers are found in all of our cases, even in case 59 on the right side, so that they are considered to arise from all of the mamillary nuclei.

The mamillary peduncle is now usually taken as a bundle of chiefly or entirely ascending fibers. However, it has been shown by many authors that it contains also a few descending fibers (Wallenberg, 1899 ; Probst, '00 ; Economo and Karplus, '10 ; van Valkenburg, '12 ; Gurdjian, '27 ; Morin, '50 ; Guillery, '57 ; Bürgi and Bucher, '60 ; Ban and Zyo, '63). Bürgi and Bucher divided the descending fibers of the mamillary peduncle into the pars descendens medialis and pars descendens lateralis. The former turns at the caudal pole of the interpeduncular nucleus toward the Gudden nucleus, while the latter rapidly spreads out laterally to pass through the substantia nigra.

In primates some authors reported the mamillopeduncular tract which consists of small fascicles of thinly medullated fibers extending from the medial mamillary nucleus to the interpeduncular nucleus. However, our experiments failed to demonstrate such fibers in the cat. Guillery ('57) also stated that there are no mamillopeduncular fibers in the rat.

The dorsal longitudinal fasciculus

The present study appears to provide new data concerning the mamillary contribution to the dorsal longitudinal fasciculus of Schütz. In all of our cases a fair number of degenerating fibers from the mamillary lesion run dorsally, partly through the periventricular gray of the posterior hypothalamus, and enter the mesencephalic central gray of the operated side, through which they descend as far caudally as the lowest level of the superior colliculus. During their course they spread laterally into the reticular formation of the mesencephalon. It is of interest to note that no essential differences are found in their distribution among all the cases studied.

According to Kölliker (1896) the fibers to the dorsal longitudinal fasciculus are accompanied by the mamillotegmental tract at the beginning of its course. Crosby and Woodburne (51) divided the thalamic portion of the dorsal longitudinal fasciculus of Schütz into 
A, B, C and D, and stated that the fascicles forming bundle D are joined by fibers from the mamillary body during their course in the hypothalamus. However, there have been no previous authors who demonstrated experimentally the fibers contributing to the dorsal longitudinal fasciculus following localized lesions in the mamillary body. Our results, particularly from cases with lesions fairly well localized in the mamillary body (cats 38, 56 and 73), indicate that the degenerating fibers in the central gray matter of the mesencephalon are derived largely from the mamillary nuclei.

\section{Summary}

1. The mamillothalamic tract arises from all of the mamillary nuclei. The pars medialis of the medial mamillary nucleus sends fibers ipsilaterally to the pars magnocellularis of the anteroventral nucleus and the anteromedial nucleus of the thalamus. Its medial portion projects to the medial portion of the pars magnocellularis of the anteroventral nucleus and the dorsomedial part of the anteromedial nucleus, while the lateral portion projects to the dorsolateral portion of the pars magnocellularis of the anteroventral nucleus. The pars lateralis of the medial mamillary nucleus sends fibers to the pars parvocellularis of the anteroventral nucleus of the same side and also to the anterodorsal nucleus of both sides. The lateral mamillary nucleus is connected with both anterodorsal nuclei.

2. The mamillary nuclei contribute fibers to the medial forebrain bundle, projecting to the medial septal nucleus. Some of these mamilloseptal fibers end in the lateral hypothalamic and the lateral preoptic nuclei on their way to the septal nucleus.

3. The mamillotegmental tract divides into the main dorsal and the ventral bundles. Although the latter is directed ventrally to the nucleus reticularis tegmenti pontis and the pontine nuclei, the former passes dorsally to the dorsal and ventral tegmental nuclei, and the central gray of the pons. The fibers from the pars medialis of the medial mamillary nucleus end chiefly in the ventral tegmental nucleus, particularly the small-celled part, but some of them run further dorsally to enter the large-celled ventral part of the dorsal tegmental nucleus. It should be mentioned that a few fibers from the pars medialis of the medial mamillary nucleus partly run dorsally along the median raphe to enter the central gray of the pons, and partly cross the midline to end in the ventral tegmental nucleus of the opposite side, though a very few fibers pass through this nucleus. The fibers from the pars lateralis of the medial mamillary nucleus are directed largely to the dorsal tegmental nucleus, particularly its 
large-celled ventral part, and partly to the ventral tegmental nucleus. Within the dorsal nucleus most fibers terminate, but some pass through it to end in the central gray of the pons. On the other hand, a few fibers from the pars lateralis of the medial mamillary nucleus run dorsally along the median raphe to terminate in the central gray of the pons, without entering the tegmental nuclei. The fibers from the lateral mamillary nucleus are distributed to the ventral and dorsal tegmental nuclei, and to the central gray of the pons along the median raphe. Besides, they are distributed to the reticular formation and the tegmental laterodorsal nucleus.

4. A small number of fibers from the mamillary nuclei descend in the mamillary peduncle and can be traced to the middle levels of the interpeduncular nucleus. During their course many of them spread dorsally to join the mamillotegmental fibers.

5. The mamillary body contributes fibers to the dorsal longitudinal fasciculus of Schütz. These fibers descend in the mesencephalic central gray of the same side as far caudally as the lowest level of the superior colliculus, spreading out laterally into the reticular formation throughout their course.

\section{Acknowledgment}

We wish to express our sincere thanks to Professor James M. Sprague, Department of Anatomy, University of Pennsylvania, for his helpful suggestions.

This study was supported in part by a grant from the Ministry of Education of the Japanese Government for Scientific Research.

\section{References}

Ban, T. and Zyo, K.: Experimental studies of the mamillary peduncle and mamillotegmental tracts in the rabbit. Med. J. Osaka Univ., 13:241-270, 1963.

Bleier, R.: Retrograde transsynaptic cellular degeneration in mamillary and ventral tegmental nuclei following limbic decortication in rabbits of various ages. Brain Research, 15 : 365-393, 1969.

Brodal, A. : Afferent cerebellar connections. In : Aspects of cerebellar anatomy. J. Jansen and A. Brodal, eds., Johan Grundt Tanum, Oslo, pp. 82-188, 1954.

Bürgi, S. M. and Bucher, V. M. : Markhaltige Faserverbindungen im Hirnstamm der Katze. Springer, Berlin, 1960.

Cajal, S. R. : Histologie du système nerveux de l'homme et des vertébrés. T. 2, A. Maloine, Paris, 1911.

Castaldi, L.: Studi sulla struttura e sullo suviluppo del mesencefalo. Ricerche in Cavia cobaya. Parte terza. Arch. ital. Anat. Embriol., 23 : 481-609, 1926.

Clark, W.E. Le Gros: An experimental study of thalamic connections in the rat. Phil. Trans. Roy. Soc. London, Ser. B, 222 : 1-28, 1933.

Cowan, W. M. and Powell, T.P.S.: An experimental study of the relation between 
the medial mamillary nucleus and the cingular cortex. Proc. Roy. Soc. London, Ser. B, 143 : 114-125, 1954.

Crosby, E.C. and Woodburne, R.T: : The mammalian midbrain and isthmus region. Part III. The fiber connections. C. The hypothalamo-tegmental pathways. J. Comp. Neur., 94 : 1-32, 1951.

Diepen, R. : Hypothalamus. Handbuch der mikroskopischen Anatomie des Menschen. Bd. 4, Teil 7, Springer, Berlin, 1962.

Economo, C. J. and Karplus, J. P.: Zur Physiologie und Anatomie des Mittelhirns. VIII. Beiträge zur Faser-Anatomie des Hirnstammes. Arch. Psychiat., 46 : $377-$ 429, 1909.

Fry, W. J. : Mamillary complex of cat brain-Aspects of quantitative organization. Anat. Rec., 154 : 175-184, 1966.

Fry, W. J. : Quantitative delineation of the efferent anatomy of the medial mamillary nucleus of the cat. J. Comp. Neur., 139:321-336, 1970.

Fry, W. J., Karmins, R., Fry, F. J., Thomas, G., Borbely, S. and Ades, H. : Origins and distributions of the some efferent pathways from the mamillary nuclei of the cat. J. Comp. Neur., $120: 195-257,1963$.

Greving, R.: Beiträge zur Anatomie des Zwischenhirns und seiner Funktion. IV. Ueber den Regulationsmechanismus der vegetativen Zentren in der Zwischenhirnbasis auf Grund zytoarchitektonischer und faseranatomischer Untersuchungen. Z. Neur., $99:$ 232-252, 1925.

Gudden, B. von: Beitrag zur Kenntnis des Corpus mamillare und der sogenannten Schenkel des Fornix. Arch. Psychiat., 11: 428-452, 1881.

Guillery, R. W. : A quantitative study of the mamillary bodies and their connections. J. Anat., $89:$ 19-32, 1955.

Guillery, R. W. : Degeneration in the hypothalamic connexions of the albino rat. J. Anat., 91 : 91-115, 1957.

Gurdjian, E. S. : The diencephalon of the albino rat. J. Comp. Neur., $43: 1-114,1927$.

Ibánez, J.S. : Étude de la dégénération du fascicule tegmental de Gudden consécutif à la lésion expérimentale du noyau mamillaire externe. Trav. Lab. rech. biol. Univ. Madrid, 30 : 211-219, 1935.

Kimoto, A. and Sakai, M. : Experimental researches on the fiber connections of the tegmental nuclei and ventral tegmental area of the midbrain (in Japanese). Acta Anat. Niigata, 49 : 63-74, 1959.

Koikegami, H. : Die Kerne und Verbindungsbahnen des Corpus mamillare der Säugetieren. Z. mikrosk.-anat. Forsch., $44: 131-162,1938$.

Kölliker, A. : Handbuch der Gewebelehre des Menschen. Bd. 2, Engelmann, Leipzig, 1896.

Krieg, W.J.S. : The hypothalamus of the albino rat. J. Comp. Neur., $55: 18-89,1932$.

Morin, F.: An experimental study of hypothalamic connections in the guinea pig. J. Comp. Neur., 92 : 193-214, 1950.

Nauta, W.J.H. : Hippocampal projections and related neural pathways to the midbrain in the cat. Brain, $81: 319-340,1958$.

Nauta, W. J.H. and Gygax, P. A. : Silver impregnation of degenerating axons in the central nervous system: A modified technic. Stain Technol., $29: 91-93,1954$.

Nauta, W. J.H. and Haymaker, W. : Hypothalamic nuclei and fiber connections. In : The hypothalamus. W. Haymaker, E. Anderson and W. J. H. Nauta, eds., C. C. Thomas, Springfield, pp. 136-209, 1969.

Niimi, K. and Kuwahara, E. : The dorsal thalamus of the cat and comparison with monkey and man. J. Hirnforsch. (in press).

Niimi, K., Nakano, T., Koizuka, M. and Ichinona, M. : Experimental-anatomical studies on the cortical connections of the anterior thalamic nuclei in carnivores. Okajimas Fol. anat. jap., 38 : 95-127, 1962. 
Powell, T.P.S. and Cowan, W.M. : The origin of the mamillo-thalamic tract in the rat. J. Anat., 88 : 489-497, 1954.

Powell, T.P.S., Guillery, R. W. and Cowan, W. M. : A quantitative study of the fornixmamillothalamic system. J. Anat., $91: 419-437,1957$.

Probst, M. : Zur Anatomie und Physiologie experimenteller Zwischenhirnverletzungen. Dtsch. Z. Nervenhk., $17: 141-168,1900$.

Rioch, D. McK. : Studies on the diencephalon of Carnivora. Part III. Certain myelinated-fiber connections of the diencephalon of the dog, cat and aevisa. $J$. Comp. Neur., 53 : 319-388, 1931.

Rose, J. : The cell structure of the mamillary body in the mammals and in man. J. Anat., 74 : 91-115, 1939.

Sprague, J. M. and Meyer, M. : An experimental study of the fornix in the rabbit. J. Anat., 84 : 354-368, 1950.

Valkenburg, C. T. van: Caudal connections of the corpus mamillare. Proc. Kon. Akad. Wet., $14:$ 1118-1121, 1912.

Votaw, C. L. : Study of septal stimulation and ablation in the macaque monkey. Neurology, $10: 202-209,1960$.

Wallenberg, A. : Notiz über einen Schleifenursprung des Pedunculus corporis mamillaris beim Kaninchen. Anat. Anz., 16: 156-158, 1899.

Yamagata, K. : Ueber die Verbindungsbahn des Corpus mamillare (in Japanese). J. Okayama Med. Soc., 39 : 614-644, 906-922, 1057-1072, 1927.

\section{Abbreviations}

Ad, anterodorsal nucleus

Am, anteromedial nucleus

Av, anteroventral nucleus

Bc, brachium conjunctivum

$\mathrm{Ca}$, anterior commissure

Cd, caudate nucleus

$\mathrm{Ci}$, internal capsule

$\mathrm{Cp}$, posterior commissure

$D$, nucleus of Darkschewitsch

$\mathrm{Db}$, diagonal band of Broca

Dt, dorsal tegmental nucleus

Dtd, dorsal tegmental nucleus, dorsal division

Dtv, dorsal tegmental nucleus, ventral division

$F$, fornix

Flm, medial longitudinal fasciculus

Fr, reticular formation

$\mathrm{Gm}$, medial geniculate body

H, field of Forel

$\mathrm{Hl}$, lateral hypothalamic nucleus

$\mathrm{Hp}$, habenulopeduncular tract

Ld, dorsal lateral nucleus

Ldt, nucleus laterodorsalis tegmenti

$\mathrm{Lm}$, medial lemniscus

M1, lateral mamillary nucleus
Mm, medial mamillary nucleus

$\mathrm{Mml}$, medial mamillary nucleus, lateral division

Mmm, medial mamillary nucleus, medial division

Pc, cerebral peduncle

Pl, lateral pontine nucleus

Pm, mamillary peduncle

Pol, lateral preoptic nucleus

$P v$, ventral pontine nucleus

$\mathrm{Rh}$, rhomboid nucleus

Rtp, nucleus reticularis tegmenti pontis

Sem, medial septal nucleus

Sgc, central gray substance

Sm, submedial nucleus

Sn, substantia nigra

Sth, subthalamic nucleus

$\mathrm{Va}$, anterior ventral nucleus

VI, lateral ventral nucleus

Vm, medial ventral nucleus

$\mathrm{Vp}$, posterior ventral nuclei

$\mathrm{Vt}$, ventral tegmental nucleus

Vtm, ventral tegmental nucleus, large-celled division

Vtp, ventral tegmental nucleus, small-celled division 
Mamillary Efferents in Cat

\section{PLATE}




\section{Explanation of Plate}

Fig. 1. Photomicrograph of a transverse section through the medial septal nucleus in cat 36 . Nauta-Gygax method- $\times 430$

Fig. 2. Photomicrograph of a transverse section through the right anterodorsal nucleus in cat 38 . Nauta-Gygax method. $\times 430$

Fig. 3. Photomicrograph of a transverse section through the left anterodorsal nucleus in cat 56 . Nauta-Gygax method. $\times 430$

Fig. 4. Photomicrograph of a transverse section through the left dorsal tegmental nucleus in cat 56 . Nauta-Gygax method. $\times 430$ 

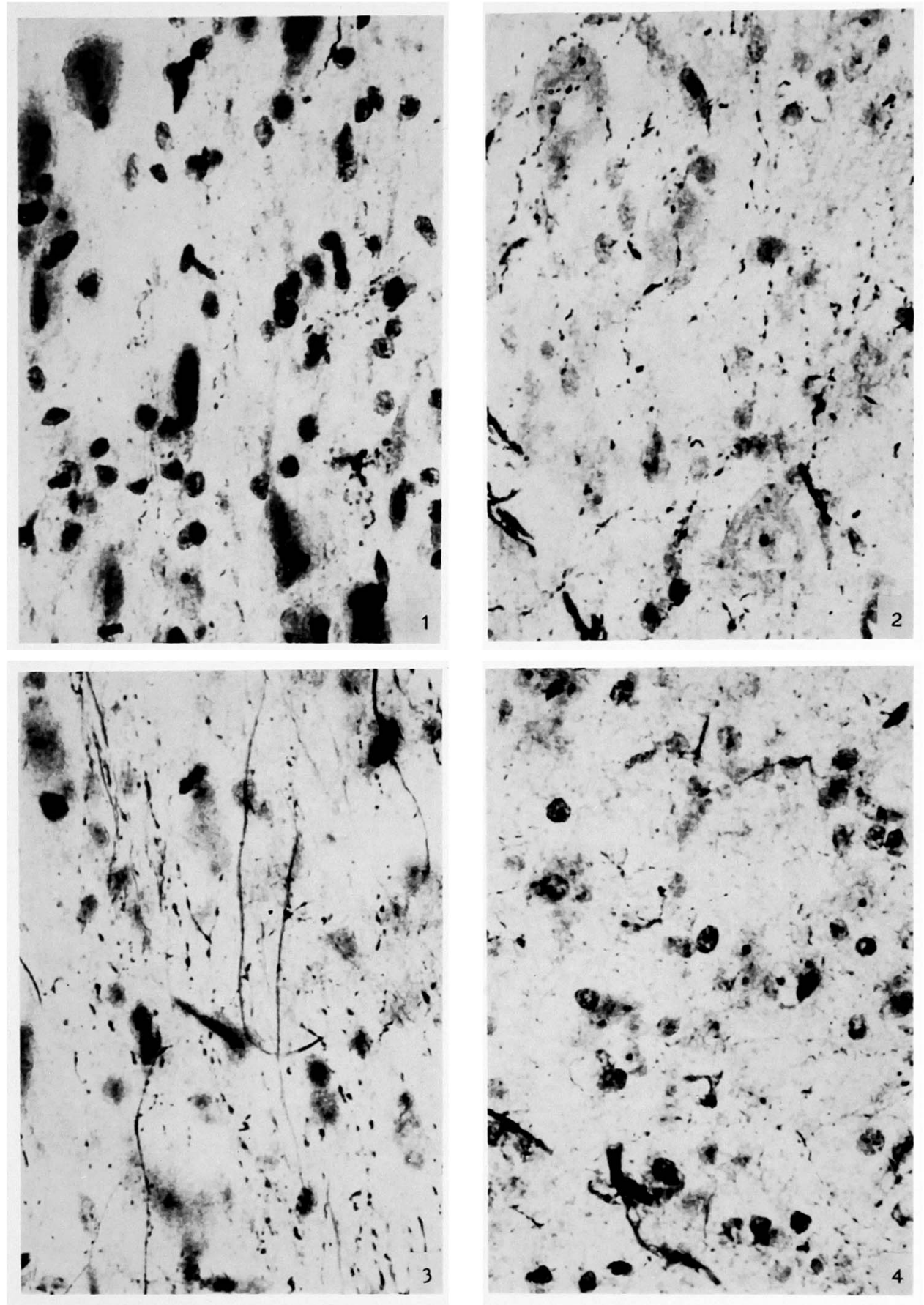